\title{
A Four-Quadrant Thrust Controller for Marine Propellers with Loss Estimation and Anti-Spin
}

\author{
Luca Pivano* Jostein Bakkeheim* Tor Arne Johansen* \\ Øyvind Notland Smogeli ${ }^{* *}$ \\ * Department of Engineering Cybernetics, Norwegian University of \\ Science and Technology, Trondheim, Norway (e-mails: [luca.pivano, \\ jostein.bakkeheim, tor.arne.johansen]@itk.ntnu.no). \\ ** Marine Cybernetics, Tiller, Norway (e-mail: \\ Oyvind.Smogeli@marinecyb.com).
}

\begin{abstract}
In this paper a nonlinear thrust controller for a fixed pitch marine propeller with torque loss estimation and an anti-spin strategy is presented. The controller, designed to work in the four-quadrant plane composed by the shaft speed and the vessel speed, is a combination of a thrust controller designed for calm sea conditions and an anti-spin strategy to reduce power peaks and wear-and-tear in extreme sea conditions. The anti-spin algorithm avoid large increase of the shaft speed once high torque losses due to ventilation are detected and set the shaft speed to normal when the loss situation is considered over. The ventilation incident is detected by monitoring the torque losses, estimated with a nonlinear observer. The performances of the proposed controller are validated by experiments carried out in a towing tank.
\end{abstract}

Keywords: Marine propulsion, thrust control, anti-spin.

\section{INTRODUCTION}

In the last years there has been a growing number of works in the design of vessel control systems focusing on the low level thrusters controller, see Whitcomb and Yoerger [1999], Fossen and Blanke [2000], Smogeli et al. [2004], Smogeli [2006], and Pivano et al. [2007]. The main difficulties to design effective propeller controllers lie in the modeling of the propeller's dynamics and in the problem of measuring the environmental state. A marine propeller is often affected by thrust losses due to inline velocity variations, ventilation, in-and-out of water effects, wave-induced water velocities, interaction between the vessel hull and the propeller and interaction between propellers. The primary objective of the low level thruster controller is to obtain the desired thrust from the propeller regardless the environmental state. The knowledge of the propeller thrust and torque, together with the thrust induced pressure force on the hull, is fundamental to achieve high vessel control performance. Unfortunately a propeller system is not usually equipped with thrust and torque sensors therefore thrust losses are not directly measured.

As reported in Smogeli et al. [2005], today's industrial standard for fixed pitch propellers is shaft speed control where the desired shaft speed is computed from the desired thrust through a static mapping. Also torque and power control and combination of those have been developed and implemented (see for example Blanke and Nielsen [1990] and Smogeli et al. [2005]). All the mentioned controllers do not use information about the propeller working condition, i.e. the controller performance may be poor when thrust losses occur.

In this paper a nonlinear thrust controller for fixed pitch propellers that includes the estimation of the propeller working condition is derived. The proposed algorithm is effective both in calm seas and in extreme sea conditions. The controller is a combination of a thrust controller for calm seas and an anti-spin strategy to reduce the power peaks and wear-and-tear in extreme sea conditions.
The thrust controller for calm seas consists of a propeller shaft speed controller where the speed reference is computed from the requested thrust to the propeller by the high level controller (e.g. a vessel speed controller) and the torque losses, estimated with a nonlinear observer. The controller is an enhancement of the one presented in Pivano et al. [2007] where the mapping to compute the desired shaft speed from the thrust reference and the torque losses has been improved. This is done in order to reduce the high control activity experienced in Pivano et al. [2007] for values of the shaft speed and the vessel speed in the $2^{\text {nd }}$ and $4^{\text {th }}$ quadrants. Moreover, in harsh sea state, high thrust losses are experienced and the controller designed in Pivano et al. [2007] increases the desired shaft speed to large values in order to counteract the losses due to ventilation. For this reason an anti-spin strategy has been implemented, reducing the shaft speed once high thrust losses due to ventilation are detected. When the ventilation is considered over, the desired shaft speed is set to normal values. The anti-spin algorithm for marine propellers was first introduced in Smogeli et al. [2003] and further developed in Smogeli et al. [2004] and Smogeli [2006]. The cited anti-spin controllers were designed for Dynamic Positioning (DP) operations where the vessel speed is small and for positive values of the shaft and vessel speed. In the present work the controller is designed for the full four-quadrant range of the propeller shaft speed and the vessel speed and also for maneuvering and transit operations where the vessel speed is larger than in DP operations.

The effectiveness of the controller has been validated by experimental tests carried out in a towing tank where the proposed scheme has been tested in simulated calm and harsh sea state.

\section{PROPELLER MODEL}

The propeller system is composed of an electric motor attached to a shaft and to a propeller through a gear-box. 
The shaft rotates at the angular speed $\omega$. Its dynamics can be written as

$$
J_{m} \dot{\omega}=Q_{m}-Q_{p}-Q_{f}(\omega),
$$

where $J_{m}$ is shaft moment of inertia, $Q_{f}(\omega)$ is the shaft friction torque which depends on the shaft speed, $Q_{m}$ is the motor torque which is controlled by the motor drive based on the reference $Q_{m_{d}}$ and $Q_{p}$ is the propeller load torque. The friction torque is modeled as a Coulomb plus a linear and nonlinear viscous effect. This model, motivated by experiments presented in Pivano et al. [2006], is written as

$$
Q_{f}(\omega)=k_{f_{1}} \frac{2}{\pi} \arctan \left(\frac{\omega}{\epsilon}\right)+k_{f_{2}} \omega+k_{f_{3}} \arctan \left(k_{f_{4}} \omega\right),
$$

where the coefficients $k_{f_{i}}$ and $\epsilon$ are constant and positive. In order to avoid the singularity in zero, the Coulomb effect, usually written as a $\operatorname{sign}(\omega)$, has been replaced by the function $\frac{2}{\pi} \arctan \left(\frac{\omega}{\epsilon}\right)$ with a small positive $\epsilon$. The propeller load torque $Q_{p}$ is represented by the torque produced at zero advance speed, that is the propeller is deeply submerged and not subjected to losses, plus a term $\Delta_{q}$ that incorporates losses of torque:

$$
Q_{p}= \begin{cases}G_{Q_{p}}|\omega| \omega+\Delta_{q} & \omega \geq 0 \\ G_{Q_{n}}|\omega| \omega+\Delta_{q} & \omega<0\end{cases}
$$

The terms $G_{Q_{p}}$ and $G_{Q_{n}}$ are positive constants and of different magnitudes due to the propeller asymmetry with respect to the shaft speed $\omega$. Neglecting the dynamics of the electrical part of the system (frequency converter, stator and rotor), usually faster than the shaft dynamics, the control input is represented by $Q_{m}=Q_{m_{d}}$. Both $Q_{m}$ and $\omega$ are measured. The term $\Delta_{q}$ is represented by a Markov like process with time constant $\tau$ driven by a bounded noise signal $w$ :

$$
\dot{\Delta}_{q}=-\frac{1}{\tau} \Delta_{q}+w \text {. }
$$

The noise $w$ represents the contribution of all the phenomena that can generate torque losses. Grouping the nonlinearities in the function $\psi(\omega)$, the system can be rewritten as:

$$
J_{m} \dot{\omega}=Q_{m}-\psi(\omega)-k_{f_{2}} \omega-\Delta_{q}
$$

where $\Delta_{q}$ is given by (4),

$\psi(\omega)=G_{Q_{p, n}}|\omega| \omega+k_{f_{1}} \frac{2}{\pi} \arctan \left(\frac{\omega}{\epsilon}\right)+k_{f_{3}} \arctan \left(k_{f_{4}} \omega\right)$,

and

$$
G_{Q_{p, n}}= \begin{cases}G_{Q_{p}}, & \omega \geq 0 \\ G_{Q_{n}}, & \omega<0 .\end{cases}
$$

A block diagram of the overall control system is shown in Fig. 1.

\section{OBSERVER FOR TORQUE LOSS ESTIMATION}

A nonlinear observer with gain $l_{1}$ and $l_{2}$ is designed to estimate the torque loss $\hat{\Delta}_{q}$ and the shaft speed $\hat{\omega}=\hat{y}$ :

$$
\begin{aligned}
J_{m} \dot{\hat{\omega}} & =Q_{m}-\psi(\hat{\omega})-k_{f_{2}} \hat{\omega}-\hat{\Delta}_{q}+l_{1}(y-\hat{y}) \\
\dot{\hat{\Delta}}_{q} & =-\frac{1}{\tau} \hat{\Delta}_{q}+l_{2}(y-\hat{y}) \\
y & =\omega .
\end{aligned}
$$

Defining the observer error variables as $\tilde{\omega}=\omega-\hat{\omega}$ and $\tilde{\Delta}_{q}=\Delta_{q}-\hat{\Delta}_{q}$, from the model in (4), (5), (6) and (7)

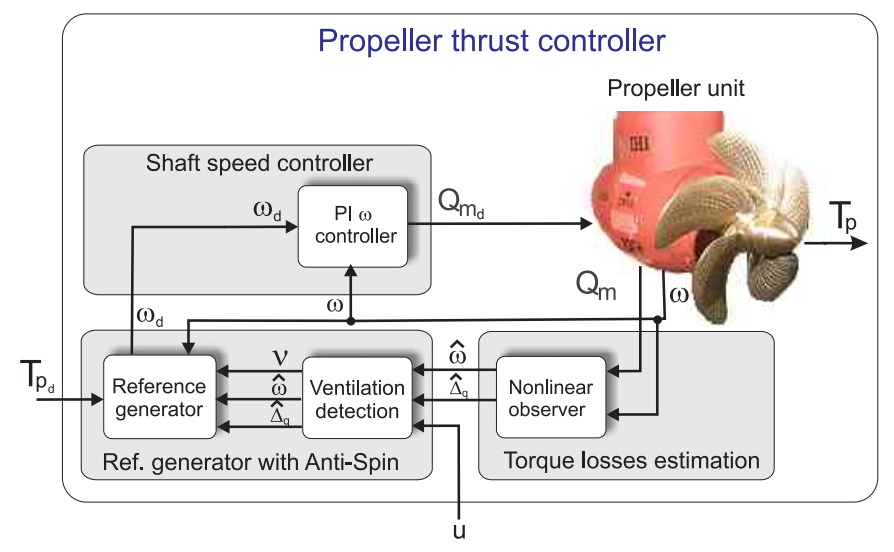

Fig. 1. Block diagram of the overall system.

and the observer in (8), (9) and (10), the observer error dynamics becomes:

$$
\begin{aligned}
J_{m} \dot{\tilde{\omega}} & =-(\psi(\omega)-\psi(\hat{\omega}))-l_{1} \tilde{\omega}-k_{f_{2}} \tilde{\omega}-\tilde{\Delta}_{q} \\
\dot{\tilde{\Delta}}_{q} & =-\frac{1}{\tau} \tilde{\Delta}_{q}-l_{2} \tilde{\omega}+w .
\end{aligned}
$$

Proposition 1. If there exist $a_{11}, a_{22}>0$ and the gains $l_{1}$ and $l_{2}$ are chosen such that

A1 $l_{1}>-k_{f_{2}}$,

$\mathbf{A 2}\left|\frac{a_{11}}{J_{m}}+a_{22} l_{2}\right|<2 \sqrt{a_{11} a_{22}\left(\frac{k_{f_{2}}+l_{1}}{\tau J_{m}}\right)}$,

then the error dynamics (11) and (12) is globally exponentially stable (GES) when $w=0 \forall t$, and input-to-state stable (ISS) with respect to $w$.

Proof. See Pivano et al. [2007].

The estimates $\hat{\omega}$ and $\hat{\Delta}_{q}$ can be used to compute an estimate of the propeller torque from

$$
\hat{Q}_{p}=G_{Q_{p, n}}|\hat{\omega}| \hat{\omega}+\hat{\Delta}_{q} .
$$

\section{THRUST CONTROLLER}

\subsection{Controller for calm sea}

We design a shaft speed controller which is derived as follows: first a desired torque $Q_{p_{d}}$ is computed from the desired thrust $T_{p_{d}}$ using the standard propeller characteristics; second a desired shaft speed is computed from $Q_{p_{d}}$ and the estimated losses $\hat{\Delta}_{q}$.

The standard propeller characteristics is measured in steady-state conditions and usually presented in the form of the non-dimensional thrust and torque coefficients $K_{T}$ and $K_{Q}$. The coefficients are plotted as a function of the advance number $J$, given from:

$$
J=\frac{2 \pi u_{a}}{\omega D},
$$

where $D$ is the propeller disc diameter and $u_{a}$ is the advance speed, i.e. the speed of the inlet water to the propeller disc. The thrust and torque coefficients are computed from Van Lammeren et al. [1969] as:

$$
\begin{aligned}
K_{T} & =\frac{4 \pi^{2} T_{p}}{\rho|\omega| \omega D^{4}}, \\
K_{Q} & =\frac{4 \pi^{2} Q_{p}}{\rho|\omega| \omega D^{5}} .
\end{aligned}
$$


Using the relations (15) and (16) in combination with the desired propeller thrust, the desired propeller torque $Q_{p_{d}}$ is computed as:

$$
Q_{p_{d}}=\frac{1}{G_{Q T}(\hat{J})} T_{p_{d}}
$$

where

$$
G_{Q T}(\hat{J})=\frac{T_{p}}{Q_{p}}=\frac{\left.K_{T}\right|_{\hat{J}}}{\left.D K_{Q}\right|_{\hat{J}}} .
$$

To compute $G_{Q T}(\hat{J})$, the value of $\hat{J}$, an estimate of the advance number, is derived employing the estimated propeller torque. Using $\hat{Q}_{p}$ instead of $Q_{p}$ in (16) we compute the estimate $\hat{K}_{Q}$. Combining the value of $\hat{K}_{Q}$ with the $K_{Q}$ curve, see Fig. 2 (a), we can derive the value of $\hat{J}$. As shown in Fig. 2 (a), the $K_{Q}$ curve is not invertible in the all plotted range. The problem of not being able to invert the $K_{Q}$ curve is solved by approximating the value of $G_{Q T}(J)$ by $G_{Q T}(0)$ in zone 1.

When the propeller works at negative values of $J\left(2^{\text {nd }}\right.$ and $4^{\text {th }}$ quadrants), the advance speed $u_{a}$ and the shaft speed $\omega$ have opposite signs. The propeller tries to reverse the inlet flow and a recirculation zone, often called a ring vortex, occurs. This is due to the interaction between the inlet flow and the reversed flow. The flow then becomes unsteady and can cause quick variations of the propeller load (Pivano et al. [2006]) and consequently oscillations of the value $K_{Q}$ and $\hat{K}_{Q}$. In this situation, even a constant value of the desired thrust could result in quick variation on the reference signal $Q_{p_{d}}$. This, in turns, may produce the shaft speed reference with high frequency content, resulting in thrust oscillations as experienced in Pivano et al. [2007]. That may cause wear-and-tear of the mechanical parts of the system. This is avoided by approximating $G_{Q T}(J)$ by a constant in zone 1 . The advance number $J$ is limited in the range $[-1.5,1.1]$ for positive $\omega$ and $[-1.5,1.0]$ for negative $\omega$, the usual working range for the tested propeller.

Figure 2 (b) shows the ratio between the propeller thrust and torque $G_{Q T}(J)$ computed from the propeller characteristics and its approximation $G_{Q T}(\hat{J})$. The plot refers to positive shaft speed $\omega$; the plot for negative speed is similar but the $K_{Q}$ and $G_{Q T}(J)$ curves present smaller magnitude due to the propeller asymmetry.

In order to track the desired propeller torque $Q_{p_{d}}$, a shaft speed controller is designed. Given the desired propeller torque $Q_{p_{d}}$, the desired shaft speed $\bar{\omega}_{d}$ is computed by inverting (3) and using the estimated torque loss $\hat{\Delta}_{q}$ :

$$
\bar{\omega}_{d}=\sqrt{\frac{\left|Q_{p_{d}}-\hat{\Delta}_{q}\right|}{G_{Q_{p, n}}}} \operatorname{sign}\left(Q_{p_{d}}-\hat{\Delta}_{q}\right) .
$$

We design a controller to track the desired shaft speed $\bar{\omega}_{d}$. To generate a smooth reference signal $\omega_{d}$ and $\dot{\omega}_{d}$, we employ a second order low pass filter with cutoff frequency equal to $\omega_{c}$ and relative damping factor $\xi$ :

$$
\ddot{\omega}_{d}+2 \omega_{c} \xi \dot{\omega}_{d}+\omega_{c}^{2} \omega_{d}=\omega_{c}^{2} \bar{\omega}_{d} .
$$

The filter is also needed because the time derivative of $\bar{\omega}_{d}$, used in the feed-forward term of the controller, is infinity when $Q_{p_{d}}-\hat{\Delta}_{q}=0$.

We employ the following control law that includes a feedforward part, a proportional action and an integral action to ensure convergence in presence of constant disturbances:

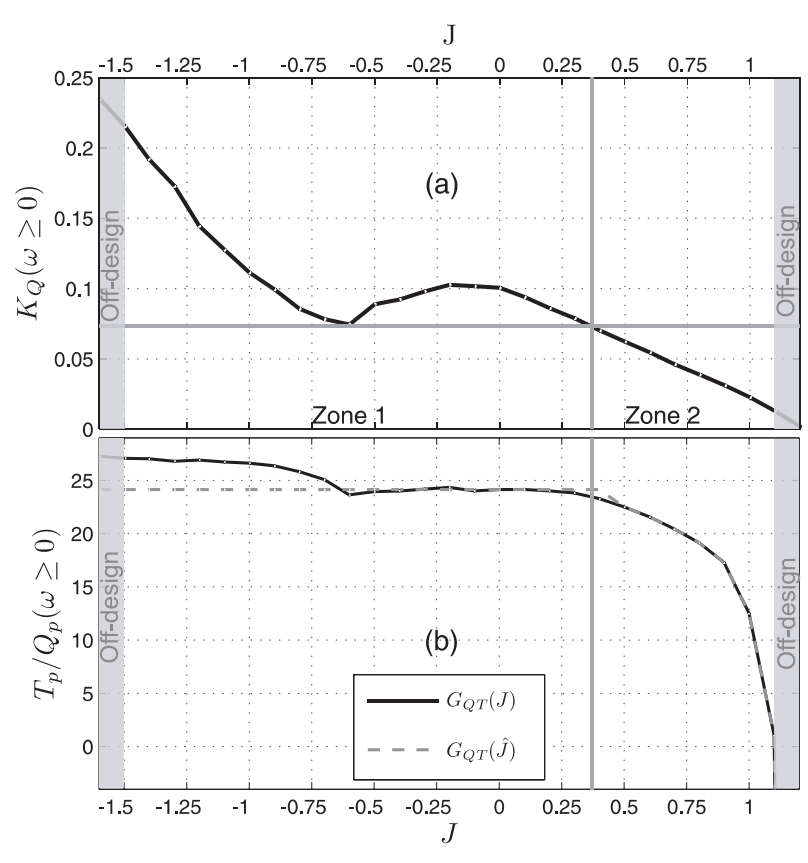

Fig. 2. $K_{Q}$ characteristic for $\omega \geq 0$ and the ratio between thrust and torque for $\omega \geq 0$.

$$
\begin{aligned}
Q_{m}= & J_{m} \dot{\omega}_{d}+\hat{\Delta}_{q}+\psi\left(-\gamma e_{1}+\omega_{d}\right)+k_{f_{2}} \omega_{d} \\
& -\left(k_{I}+\gamma k_{P}\right) e_{1}-k_{P} e_{2} .
\end{aligned}
$$

Defining the control error $e_{1}=\int_{0}^{t}\left(\omega(\tau)-\omega_{d}(\tau)\right) d \tau$ and $e_{2}=\omega-\omega_{d}$, the control error dynamics becomes:

$$
\begin{aligned}
\dot{e}_{1}= & e_{2} \\
\dot{e}_{2}= & -\frac{k_{f_{2}}}{J_{m}} e_{2}-\frac{1}{J_{m}}\left[\psi(\omega)-\psi\left(-\gamma e_{1}+\omega_{d}\right)\right]-\frac{k_{P}}{J_{m}} e_{2} \\
& -\frac{1}{J_{m}}\left(k_{I}+\gamma k_{P}\right) e_{1}-\frac{1}{J_{m}} \tilde{\Delta}_{q} .
\end{aligned}
$$

Proposition 2. If the gains $\gamma, k_{I}$ and $k_{P}$ are chosen such that

B1 $\gamma>0$,

B2 $k_{P}>0, k_{P}>\gamma J_{m}-k_{f_{2}}$,

B3 $k_{I}>0, k_{I}>-J_{m} \gamma^{2}+\gamma k_{f_{2}}$,

then the origin of the overall error dynamics (observer + controller) is ISS with respect to $w$.

Proof. See Pivano et al. [2007].

Furthermore, as proved in Pivano et al. [2007], the bound of the control error decreases by increasing the control gains. The resulting propeller thrust is bounded and converges to a ball around the desired thrust.

\subsection{Anti-Spin Strategy}

When the propeller is deeply submerged, losses of thrust are mainly caused by variations of advance speed, interaction between the propeller and the vessel hull or between propellers and cross flows. The losses sensed through the torque loss observer are compensated by the controller by changing the shaft speed to fulfill the required thrust. When the propeller spins close to the water surface the shaft load decreases due to ventilation and a drop of thrust and torque occurs, as documented by experimental test in Pivano et al. [2006] and Bakkeheim et al. [2006]. When the ventilation incident occurs high thrust losses 
are experienced and the calm sea controller, in order to compensate them, increases the value of desired shaft speed to large values. In Smogeli et al. [2003] it has also been experimentally demonstrated that during ventilation a reduction of the shaft speed may increase the propeller thrust. These considerations motivate the use of an antispin strategy that also reduces the power peaks and wearand-tear of the mechanical parts due to large shaft speeds. The anti-spin algorithm avoids an excessive increase of the shaft speed when high torque losses are detected by setting desired shaft speed to a constant value. When the loss situation is considered over the desired shaft speed is set to normal values.

Ventilation Detection The ventilation incident is detected monitoring the ratio between the estimated propeller torque given by (13) and the nominal torque $Q_{p_{n}}$ computed from the $K_{Q}$ coefficient through (16). The ratio, often termed as the torque reduction coefficient, is written as

where

$$
\hat{\beta}_{Q}=\alpha(\omega)+(1-\alpha(\omega)) \frac{\hat{Q}_{p}}{Q_{p_{n}}},
$$

and

$$
\alpha(\omega)=e^{-k|\omega|^{p}}
$$

$$
Q_{p_{n}}=K_{Q} \frac{\rho|\omega| \omega D^{5}}{4 \pi^{2}} .
$$

The weighting function $\alpha(\omega)$ with positive tuning gains $k$ and $p$ is used to avoid the singularity of $\hat{Q}_{p} / Q_{p_{n}}$ at $\omega=0$. The nominal value of $K_{Q}$ in (25) is derived from the $K_{Q}$ characteristic using the nominal value of $J$ computed from (14) using the steady-state relation

$$
u_{a}=\left(1-w_{f}\right) u
$$

where $\left|w_{f}\right|<1$ is the wake fraction number, often identified from experimental tests, and $u$ is the vessel speed. The wake fraction number accounts for the reduction of the water velocity to the propeller caused by the vessel hull.

The ventilation is detected when the value of $\hat{\beta}_{Q}$ becomes smaller than a threshold value $\beta_{v, \text { on }}$. It is considered terminated when the value of $\hat{\beta}_{Q}$ becomes larger than $\beta_{v, \text { off }}$. The ventilation state, described by the variable $\nu$, is defined as follows:

$$
\begin{aligned}
& \hat{\beta}_{Q} \geq \beta_{v, \text { on }} \quad \Rightarrow \quad \nu=0, \text { no ventilation } \\
& \hat{\beta}_{Q}<\beta_{v, \text { on }} \Rightarrow \nu=1, \text { ventilation on } \\
& \hat{\beta}_{Q} \geq \beta_{v, \text { off }} \Rightarrow \nu=0, \text { ventilation off }
\end{aligned}
$$

The hysteresis in the ventilation detection is included in order to increase its robustness with respect to measurement noise that could affect the $\hat{\beta}_{Q}$ estimate. When the propeller works in the $2^{\text {nd }}$ or $4^{\text {th }}$ quadrant of the plane $\left(u_{a}, \omega\right)$, the nominal torque model (25) may be inaccurate (see Pivano et al. [2006] for example) leading to jumps in the ventilation state. For this reason a ventilation detection is held on for a minimum time $\mathrm{T}_{\text {hold }}$.

Anti-spin Action When the ventilation is detected, the desired shaft speed is defined as follows:

$$
\omega_{d}= \begin{cases}\omega_{v_{o p t}} & \text { if } \nu=1 \text { and } \omega_{d} \geq \omega_{v_{o p t}} \\ \omega_{d} & \text { otherwise }\end{cases}
$$

The value of $\omega_{v_{o p t}}$ is chosen such that the thrust produced by the propeller at $\omega_{v_{o p t}}$ is equal or larger than for higher shaft speeds. It may also be chosen according to an optimization criterion based on the estimate of torque loss.
Other criteria may include power peaks and the torque oscillations. This is subject to further research.

\section{EXPERIMENTAL RESULTS}

The tests were performed at the MCLab, an experimental laboratory equipped with a towing carriage that can reach a maximum speed of $2 \mathrm{~m} / \mathrm{s}$. The rig with motor, underwater housing, shaft and propeller was attached to the towing carriage in order to move it through the water. The propeller was a fixed pitch propeller with diameter $25 \mathrm{~cm}$. The real-time system Opal RT-Labß) was used

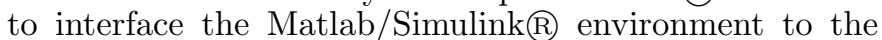
motor drive and the sensors. We employed a three phase brushless motor commanded by a drive equipped with a built-in torque controller sufficiently fast to consider its dynamics neglectable. The thrust was measured with an inductive transducer and the torque was measured with a strain gauge transducer placed on the propeller shaft.

The results reported describe one experiment carried out in calm water (to reproduce calm sea conditions) and two conducted in order to reproduce the motion that the propeller may experience in harsh sea conditions. In all three tests the propeller works in the all four quadrants of the plain $\left(u_{a}, \omega\right)$. In order to simulate a realistic scenario the towing carriage speed is first positive when the thrust is positive and then become negative when the thrust is reversed. The advance speed $u_{a}$ is considered equal to towing carriage speed since the propeller housing does not create a significant wake. The measured propeller thrust and torque, the shaft speed, the motor power and the submergence signals have been filtered with a low pass filter with cut-off frequency of $10 \mathrm{~Hz}$.

Figure 3 shows data from the calm sea test where the produced thrust $T_{p}$ tracks well the desired one $T_{p_{d}}$ despite the changes in the advance speed. Also the shaft speed tracking error is very small. The highest errors occur when reversing the thrust. The flow around the propeller becomes irregular and very fast changes in the torque losses are not captured by the observer. The estimated torque and the measured one are not presented due to lack of space. The commanded motor torque $Q_{m_{d}}$ is depicted in Fig. 3 (e).

Figure 4 is relative to the test where the propeller was also moved along its vertical axis. This is done in order to reproduce the flow variations that a propeller may encounter in harsh sea state due to the motion of the vessel when traveling in waves. In this test, the antispin algorithm was employed. The value of $\omega_{v_{\text {opt }}}$ has been set equal to $45 \mathrm{rad} / \mathrm{s}$ for positive $\omega$ and $54 \mathrm{rad} / \mathrm{s}$ for negative $\omega$. This choice is done since the produced thrust is asymmetric with respect to the shaft speed. In Fig. 4 (a) the measured propeller torque and its estimate are plotted showing good agreement. Part (b) of the same figure shows the torque loss reduction used for the ventilation detection. The ventilation state is plotted in Fig. 4 (c) together with the propeller submergence. The propeller submergence $h$ is defined equal to zero when the center of the propeller is at the water level and positive when the propeller is submerged. The ventilation incident is detected when the propeller moves toward the water surface and the propeller torque has a drop. In Fig. 4 (e) we can notice that before the ventilation is detected, the controller increases the shaft speed to compensate for the torque loss but when the ventilation is detected the desired speed is set to the $\omega_{v_{\text {opt }}}$ value. Also in this situation, despite the shaft load variation, the shaft speed controller furnishes very good performances.

Figure 5 shows data from a test similar to the second one where the anti-spin algorithm was disabled. During the 


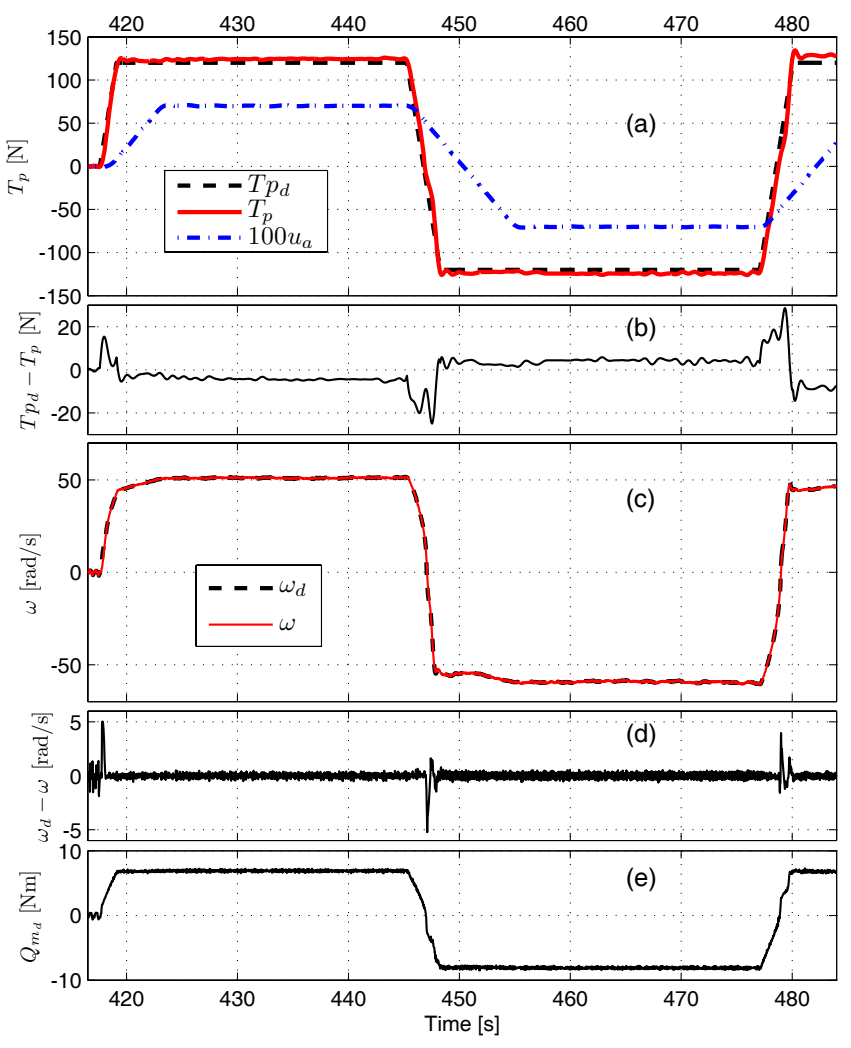

Fig. 3. Undisturbed water test: propeller thrust and advance speed (a), thrust error (b), shaft speed (c), shaft speed error (d) and desired motor torque (e).

ventilation incident the shaft speed increases quickly to the maximum value which was set to $75 \mathrm{rad} / \mathrm{s}$. Comparing these results with the test employing the anti-spin, we can notice that without anti-spin the propeller thrust fluctuates more and, even if the shaft speed is almost double, the produced thrusts when the propeller rotates close to the water surface is practically equal to the one obtained when the anti-spin is enabled. Figure 6 shows the measured motor power $P_{m}$ for the three tests. The controller without anti-spin presents large power peaks that may not be tolerated by the power generators. Also, without employing the anti-spin strategy, the mean power consumption is larger. Tab. 1 presents the mean values of the propeller thrust and the consumed power and an energy efficiency number. The mean thrust produced without the anti-spin strategy is slightly larger than with the anti-spin. This is due to the thrust spikes that occur when the ventilation incident is terminating, where the shaft speed is at the maximum value and the propeller submergence increases toward deeply submerged values. The energy efficiency number is given by the ratio between the mean power generated by the propeller and the mean motor power:

$$
\eta_{E}=\frac{\operatorname{mean}\left(T_{p} \cdot u_{a}\right)}{\operatorname{mean}\left(P_{m}\right)}
$$

The controller with anti-spin increases the energy efficiency of about $20 \%$ with respect the case where the antispin is not employed.

Table 1. Mean thrust and power and efficiency number.

\begin{tabular}{|l|c|c|c|}
\hline Controller & $T_{p}[N]$ & $P_{m}[W]$ & $\eta_{E}$ \\
\hline Anti-spin & 79.2 & 290 & 0.151 \\
No anti-spin & 89.1 & 378 & 0.123 \\
\hline
\end{tabular}

\section{CONCLUSION}

In this paper a nonlinear thrust controller for a fixed pitch marine propeller with torque loss estimation and anti-spin strategy has been presented. The controller is able to work in the four-quadrant plane composed by the shaft speed and the vessel speed and is a combination of a thrust controller designed for calm seas and an antispin strategy to reduce power peaks and wear-and-tear in extreme sea conditions. The anti-spin algorithm lowers the shaft speed once high torque losses due to ventilation are detected and increases the shaft speed to normal when the loss situation is considered over. The ventilation incident is detected by monitoring the torque losses, estimated with a nonlinear observer. Experiments showed that when large thrust losses occur, the controller that employs the anti-spin algorithm increases the energetic efficiency and reduces the power fluctuation with respect the controller without anti-spin.

\section{ACKNOWLEDGMENTS}

The Research Council of Norway is acknowledged as the main sponsor of this project.

\section{REFERENCES}

J. Bakkeheim, Ø. N. Smogeli, T. A. Johansen, and A. J. Sørensen. Improved Transient Performance by Lyapunov-based Integrator Reset of PI Thruster Control in Extreme Seas. In 45th IEEE Conference on Decision and Control, San Diego, CA, USA, 13-15 December 2006.

M. Blanke and P. Busk Nielsen. The Marine Engine Governor. In 2nd Int. Conf. on Maritime Communications and Control, pages 11-20, London, UK, 1990.

T. I. Fossen and M. Blanke. Nonlinear output feedback control of underwater vehicle propellers using feedback from estimated axial flow velocity. IEEE Journal of Oceanic Engineering, 25(2):241-255, April 2000.

L. Pivano, Ø. N. Smogeli, T. A. Johansen, and T. I. Fossen. Experimental Validation of a Marine Propeller Thrust Estimation Scheme. In 7th IFAC Conference on Manoeuvring and Control of Marine Craft (MCMC), Lisbon, Portugal, September 2006.

L. Pivano, T. A. Johansen, Ø. N. Smogeli, and T. I. Fossen. Nonlinear Thrust Controller for Marine Propellers in Four-Quadrant Operations. In 26th American Control Conference (ACC07), New York, USA, July 2007.

Ø. N. Smogeli. Control of Marine Propellers: From Normal to Extreme Conditions. PhD thesis, Department of Marine Technology, Norwegian University of Science and Technology (NTNU), Trondheim, Norway, September 2006.

Ø. N. Smogeli, L. Aarseth, E. S. Overå, A. J. Sørensen, and K. J. Minsaas. Anti-Spin Thruster Control in Extreme Seas. In 6th IFAC Conf. on Manoeuvring and Cont. of Marine Craft (MCMC03), page 221226, Girona, Spain, 2003.

Ø. N. Smogeli, J. Hansen, A. J. Sørensen, and T. A. Johansen. Anti-spin control for marine propulsion systems. In 43 rd IEEE Conference on Decision and Control, Paradise Island, Bahamas, 14-17 December 2004.

$\varnothing$ Ø. N. Smogeli, E. Ruth, and A. J. Sorensen. Experimental validation of power and torque thruster control. In IEEE 13th Mediterranean Conference on Control and Automation (MED'05), pages 1506-1511, Cyprus, June 2005.

W. P. A. Van Lammeren, J. D. Van Manen, and M. W. C. Oosterveld. The Wageningen B-Screw Series. Transactions of SNAME, 1969.

L. L. Whitcomb and D. Yoerger. Developement, comparison, and preliminary experimental validation of nonlinear dynamic thruster models. IEEE Journal of Oceanic Engineering, 24(4):481-494, Oct. 1999. 


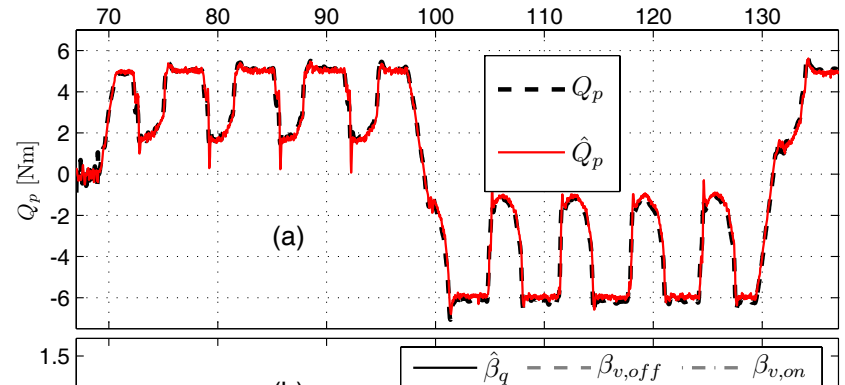

(b)
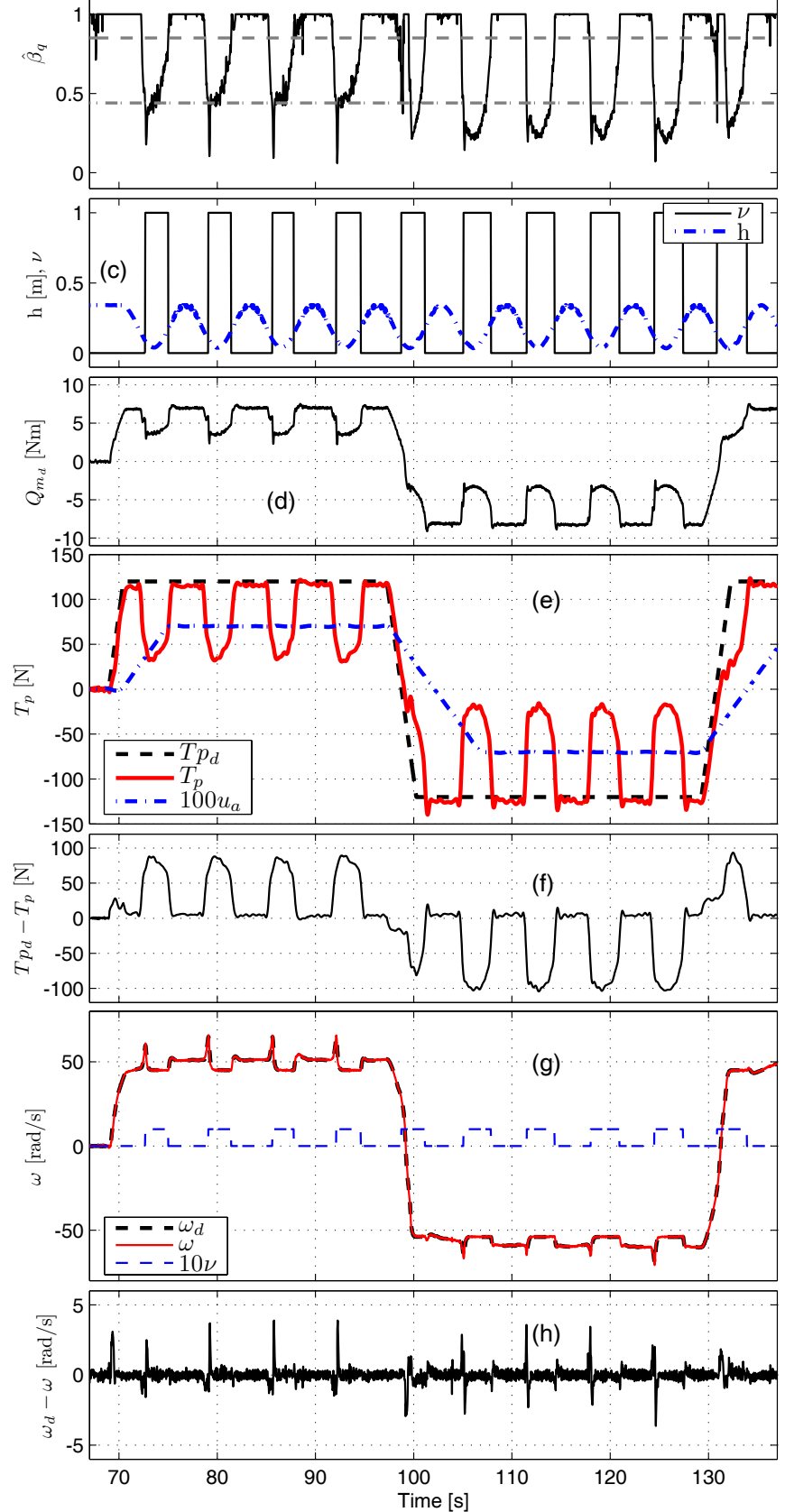

Fig. 4. Test with large thrust losses and anti-spin: propeller torque and its estimate (a), ventilation detection (b) and (c), desired motor torque (d), propeller thrust and advance speed (e), thrust error (f), shaft speed (g) and shaft speed error (h).
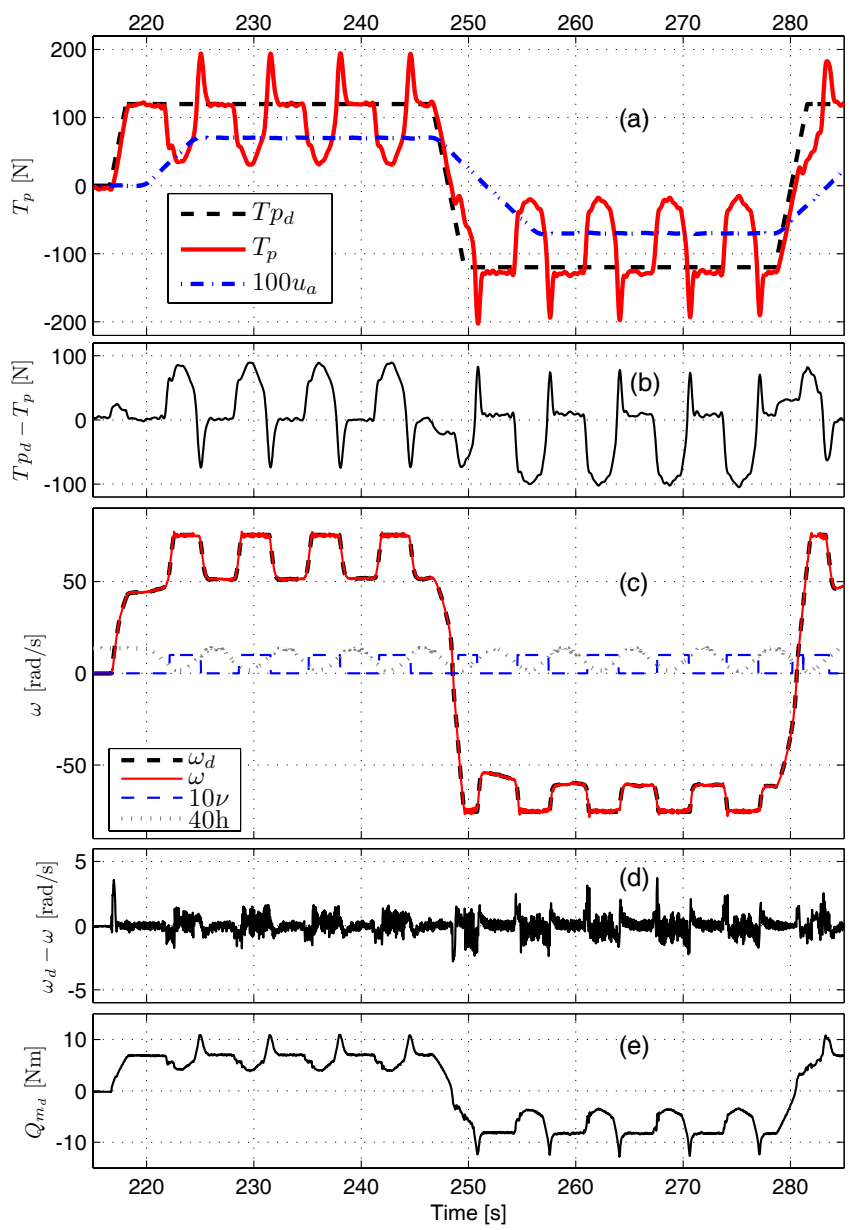

Fig. 5. Test with large thrust losses and without anti-spin: propeller thrust and advance speed (a), thrust error (b), shaft speed (c), shaft speed error (d) and desired motor torque (e).
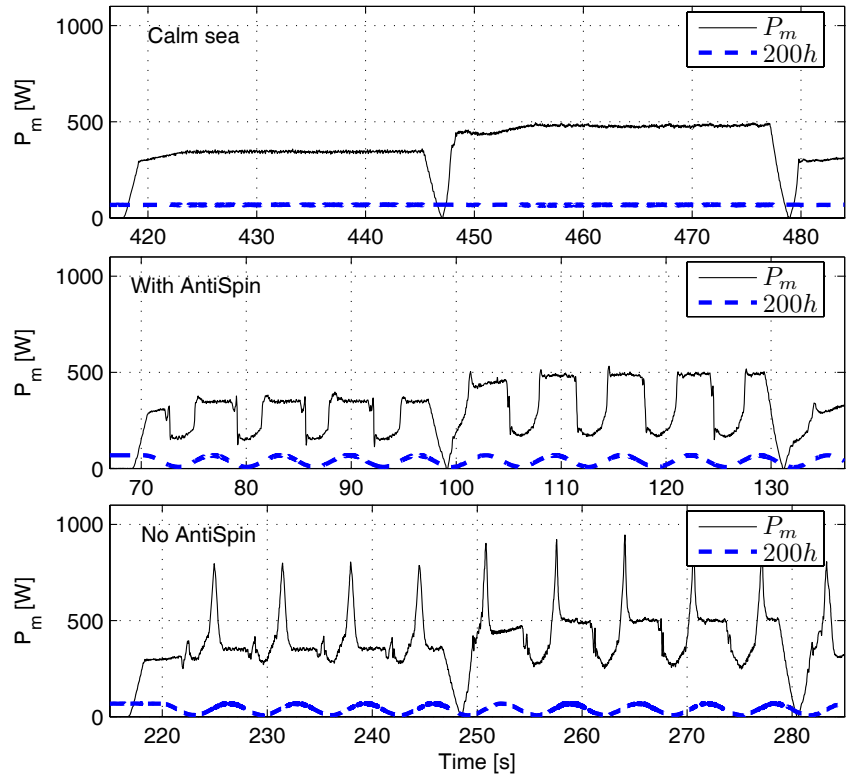

Fig. 6. Motor power in calm water and with large thrust losses (with and without the anti-spin algorithm). 